\title{
Calibração do índice de conforto para espaços externos Physiological Equivalent Temperature (PET) para Curitiba
}

\author{
Calibration of the outdoor comfort index Physiological \\ Equivalent Temperature (PET) for Curitiba
}

\section{Eduardo Leite Krüger \\ Francine Aidie Rossi \\ Pablyne Sant'Ana Cristeli \\ Henor Artur de Souza}

\section{Resumo \\ A}

análise da relação entre conforto térmico e as condições climáticas de determinada localidade pode ser uma importante ferramenta de planejamento urbano. Uma das formas de avaliação é a utilização de índices de conforto, como, por exemplo, o PET (Physiological Equivalent Temperature), índice amplamente utilizado em estudos de conforto térmico em espaços abertos, contudo apenas após a devida calibração às preferências térmicas da população local. Este trabalho teve como objetivo apresentar uma calibração do índice PET para Curitiba, PR, cidade de clima temperado de altitude (Cfb), por três procedimentos distintos. Os dados climáticos e subjetivos foram obtidos em campanhas de monitoramento térmico entre 2009 e 2010, que abrangeram verão, outono e inverno. Para a calibração, foram utilizados três métodos, notadamente: (a) agrupamento dos votos de percepção térmica a partir de entrevistados para variações discretas de $1^{\circ} \mathrm{C}$ na escala PET; (b) identificação de categorias mais frequentes para votos agrupados de percepção térmica, por mesma variação de grau PET; e (c) método das curvas Probit. O segundo método foi considerado pouco preciso na definição dos limites para Curitiba, enquanto o método Probit gerou resultados semelhantes aos obtidos pelo primeiro método, a partir dos quais foi estabelecida uma faixa de conforto térmico para espaços externos.

Palavras-chave: Índice PET. Calibração. Índice de conforto em espaços abertos. Probit.

Eduardo Leite Krüger Universidade Tecnológica Federal do Paraná Curitiba - PR - Brasil

Francine Aidie Rossi Universidade Federal do Paraná Curitiba - PR - Brasil

Pablyne Sant'Ana Cristeli Universidade Federal de Ouro Preto Ouro Preto - MG - Brasil

Henor Artur de Souza Universidade Federal de Ouro Preto Ouro Preto - MG - Brasil

Recebido em 22/11/17 Aceito em 09/02/18

\section{Abstract}

The analysis of the relationship between thermal comfort and the climatic conditions of a given location can be an important tool for urban planning. A possible way to perform such evaluation is by means of comfort indices, such as PET (Physiological Equivalent Temperature), which is widely used in thermal studies in open-air environments, after duly calibration to local thermal preferences. This study aims to calibrate the PET index for Curitiba, a city characterized by a temperate climate (Cfb), according to three distinct procedures. Climatic and subjective data were obtained in outdoor thermal monitoring campaigns during 2009 and 2010, covering summer, autumn and winter. Calibration procedures involved three distinct methods: 1) binning method for 1 ${ }^{\circ} \mathrm{C}$ discrete variations in the PET scale; 2) identifying the most prevailing thermal sensation categories in each $1{ }^{\circ} \mathrm{C}$ PET-bin; 3) Probit analysis. Method 2 was considered less precise in defining limits for Curitiba while Probit results corresponded well with those from Method 1, by which a comfort zone for outdoor spaces was obtained.

Keywords: PET index. Calibration. Outdoor comfort index. Probit.

KRÜGER, E. L.; ROSSI, F. A.; CRISTELI, P. S. SOUZA, H. A. de. Calibração do índice de conforto para espaços externos 135 Physiological Equivalent Temperature (PET) para Curitiba. Ambiente Construído, Porto Alegre, v. 18, n. 3, p. 135-148, jul./ set. 2018. 


\section{Introdução}

O uso de índices de conforto térmico auxilia no entendimento da percepção térmica dos usuários de espaços abertos. O PET (Physiological Equivalent Temperature) é um índice baseado no balanço térmico do corpo, o qual indica os efeitos do ambiente térmico em termos de estresse térmico para calor/frio e conforto. O desenvolvimento de índices com base no balanço térmico, que considerem fatores climáticos e fisiológicos, permite uma análise mais detalhada da interação entre a percepção térmica e as condições climáticas do ambiente analisado (MATZARAKIS; BLAZEJCZYK; AMELUNG, 2007; ASSOCIATION..., 2008).

Uma das vantagens do índice PET é que as variáveis climáticas são as mesmas utilizadas em outros índices de conforto, o que torna possíveis análises comparativas. Assim como o PMV (Predicted Mean Vote) (FANGER, 1970), o PET utiliza as variáveis climáticas temperatura do ar, umidade relativa, velocidade do ar e temperatura radiante média para estimar a sensação térmica.

O PET é um índice baseado no modelo MEMI (Munich Energy-balance Model for Individuals), que tem como base o balanço de energia de um indivíduo. É,

\section{[...] um índice para cálculo do conforto térmico, adaptado às condições externas, que considera não só a temperatura, umidade e vento, mas também a temperatura radiante média e é baseado na equação de equilíbrio térmico humano em estado de uniformidade [...]. (MAYER; HÖPPE, $1987^{1}$, apud LOIS; LABAKI, 2001).}

Os valores estimados são expressos em graus Celsius e são definidos como a temperatura fisiológica equivalente, em determinado ambiente (interno ou externo), à temperatura do ar de um ambiente interno de referência, no qual o balanço térmico humano é mantido com as temperaturas da pele e do centro do corpo iguais àquelas das condições em avaliação (HÖPPE, 1999). Esse índice é um dos recomendados pela Associação Alemã de Engenheiros (Verein Deutscher Ingenieure - Association of German Engineers (2008)) como parte da regulamentação para o planejamento urbano e regional.

Vários estudos utilizaram o PET para analisar a relação entre a influência do desenho urbano na sensação térmica, tais como os de Ali-Toudert e Mayer (2006), Johansson (2006), Johansson e

IMAYER, H.; HÖPPE, P. Thermal comfort of man in different urban environments. Theoretical and Applied Climatology, $v$ 38, p. 43-49, 1987.
Emmanuel (2006), Thorsson et al. (2007), Makaremi et al. (2011), Rossi, Krüger e Bröde (2011) e Rossi, Krüger e Nikolopoulou (2011). No entanto, embora Mayer e Höppe ( $1987^{2}$ apud LOIS; LABAKI, 2001) tenham proposto faixas de temperatura para o índice PET correspondentes a diferentes sensações térmicas humanas, o uso do índice em diversas localidades mostrou variações nas preferências térmicas da população local ante os valores de referência quanto à sensação térmica.

De acordo com Salata et al. (2016), há grande diversidade climática, de latitude e de continentes nos quais estudos de conforto térmico foram conduzidos na última década, variando de $1,3{ }^{\circ} \mathrm{N}$ (Manado, Indonésia) a 55,9 ${ }^{\circ} \mathrm{N}$ (Glasgow, Reino Unido). Assim, a calibração de índices de conforto térmico em espaços abertos deve ser considerada como relevante para refletir de fato as preferências térmicas locais. Como exemplos de calibração do índice PET há os trabalhos de Monteiro e Alucci (2006), que calibraram o PET para a cidade de São Paulo; Lin e Matzarakis (2008), para Sun Moon Lake, em Taiwan; Lai et al. (2014), para Tianjin, China; Hirashima et al. (2015), que calibraram o PET para duas diferentes regiões climáticas, Belo Horizonte (clima tropical) e Kassel e Freiburg na Alemanha (clima temperado); Silva e Alvarez (2015), para Vitória, ES; Salata et al. (2016), para Roma, Itália; e Lucchese et al. (2016), para Campo Grande, MS.

As pesquisas citadas utilizam diferentes métodos de calibração, o que indica que não há consenso sobre qual estratégia utilizar. Assim, além de populações provenientes de diferentes contextos climáticos e culturais apresentarem exigências térmicas distintas, temperaturas neutras e faixas de conforto/estresse térmico resultam muitas vezes de diferentes metodologias, o que dificulta uma comparação direta entre estudos. A revisão feita por Salata et al. (2016) mostra que, entre os estudos analisados, há diferenças nos métodos de análise, números de situações urbanas analisadas, número de medições, épocas do ano, horas do dia, características da população analisada, entre outros. Essa diversidade reforça o argumento de Johansson et al. (2014) sobre a necessidade de padronização dos protocolos e métodos para pesquisas de conforto em espaços abertos.

Pantavou et al. (2014) apresentaram em recente artigo uma comparação entre diferentes métodos de calibração envolvendo diversos índices térmicos em

2MAYER, H.; HÖPPE, P. Thermal comfort of man in different urban environments. Theoretical and Applied Climatology, $v$ 38, p. 43-49, 1987. 
zonas climáticas mediterrâneas. A pesquisa envolveu coletas in loco com aplicação de questionários de conforto, utilização de métodos de regressão linear e cúbica, assim como regressão Probit, com minuciosa comparação estatística das zonas de conforto encontradas (rho de Spearman, medida de associação por simetria Gamma e percentual de votos corretamente alocados). No caso do índice PET, a regressão Probit mostrou-se ligeiramente vantajosa em relação à linear, com diferenças pouco significativas na calibração desse índice.

O objetivo deste artigo é apresentar resultados de três procedimentos de calibração do índice PET para Curitiba, definindo-se a partir de suas respostas uma zona de conforto calibrada para esse índice como suporte a pesquisas futuras de conforto em espaços abertos.

\section{Método}

A calibração foi realizada a partir de dados obtidos em estudo de campo, o qual envolveu intensivas campanhas de monitoramento térmico urbano ao longo do período entre janeiro de 2009 e agosto de 2010, abrangendo, assim, verão, outono e inverno (ROSSI, 2012).

\section{Levantamento de campo das variáveis climáticas e pessoais}

Os levantamentos de campo ocorreram entre 10h e $15 \mathrm{~h}$ em ruas de pedestres e/ou de acesso motorizado limitado na região central da cidade (Figura 1). O horário foi padronizado de forma a evitar períodos de trânsito intraurbano de pessoas, compreendendo uma faixa horária durante a qual os transeuntes já estariam aclimatados à região estudada. Foram realizados 15 levantamentos de campo entre janeiro de 2009 e agosto de 2010, tendo sido monitorados dois pontos por campanha para um conjunto de 18 pontos levantados. Os pontos foram escolhidos em função de suas características morfológicas, tanto para subsidiar estudos complementares quanto a esta inter-relação, quanto para que a amostra fosse diversificada em termos de contexto urbano. Ao longo das campanhas foram monitorados dados climáticos de acordo com a ISO 7726 (INTERNATIONAL..., 1998) por duas estações meteorológicas HOBO (Onset Computer), equipadas com um anemômetro giratório a aproximadamente 2,1 $\mathrm{m}$ de altura; sensores de temperatura do ar, umidade relativa e um termômetro de globo na cor cinza a 1,1 m de altura; e um piranômetro a 1,6 m de altura. Além das variáveis de conforto, tais como temperatura do ar, umidade relativa, velocidade do ar e radiação solar, foi obtida, posteriormente, a temperatura radiante média, Trm, a qual se calculou a partir da equação para convecção forçada constante da ISO 7726 (INTERNATIONAL..., 1998). As características dos sensores usados nas campanhas estão representadas na Tabela 1.

A classificação climática de Köppen-Geiger para Curitiba é Cfb (clima temperado úmido com verão temperado, em elevação). Sua situação climática particular em relação ao conjunto de climas brasileiros e de zonas bioclimáticas apresenta desafios para urbanistas locais e poucos pontos de referência na literatura técnico-científica nacional quanto ao traçado urbano e/ou delineamento de equipamentos urbanos com função de adequação bioclimática.

\section{Figura 1 - Pontos monitorados em Curitiba}

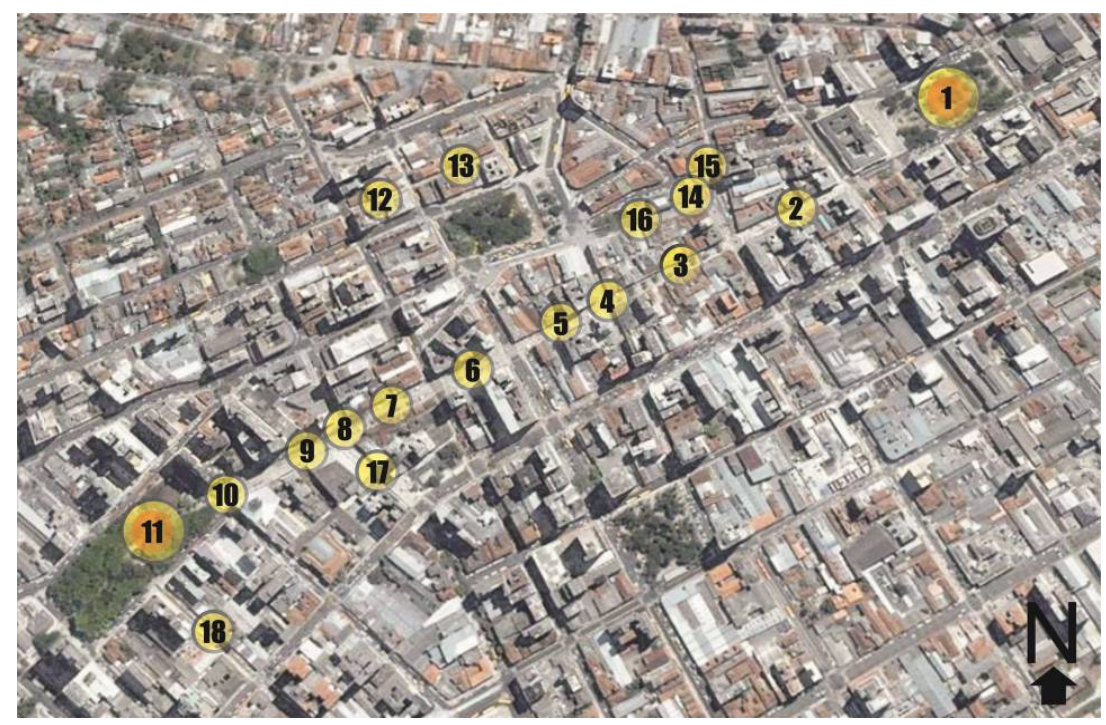


Tabela 1 - Características dos sensores utilizados nas medições

\begin{tabular}{c|c|c|c}
\hline Sensor & $\begin{array}{c}\text { Resolução por } \\
\text { unidade de medida }\end{array}$ & $\begin{array}{c}\text { Intervalo de } \\
\text { medição }\end{array}$ & Precisão \\
\hline Temperatura do ar & $0,02{ }^{\circ} \mathrm{C}$ & $-40{ }^{\circ} \mathrm{C} \mathrm{a}+75^{\circ} \mathrm{C}$ & $\pm 0,2^{\circ} \mathrm{C}$ entre $0{ }^{\circ} \mathrm{C} \mathrm{e} 50^{\circ} \mathrm{C}$ \\
\hline Umidade do ar & $0,1 \%$ & 0 a $100 \%$ & $\pm 2,5 \%(0-90 \%), \pm 3,5 \%(90-100 \%)$ \\
\hline & & 1 a $80 \mathrm{~m} / \mathrm{s}$ & $\pm 0,5 \mathrm{~m} / \mathrm{s}$ \\
Velocidade do ar & $0,4 \mathrm{~m} / \mathrm{s}$ & $\begin{array}{c} \pm 3 \% \text { entre } 17 \text { e } 30 \mathrm{~m} / \mathrm{s} \\
\pm 4 \% \text { entre } 30 \text { e } 44 \mathrm{~m} / \mathrm{s}\end{array}$ \\
\hline Direção do vento & $0,19 \mathrm{~m} / \mathrm{s}$ & 0 a $44 \mathrm{~m} / \mathrm{s}$ & $\pm 3{ }^{\circ}$ \\
\hline Radiação solar & $1,25 \mathrm{~W} / \mathrm{m}^{2}$ & 0 a $1.280 \mathrm{~W} / \mathrm{m}^{2}$ & $\pm 10 \mathrm{~W} / \mathrm{m}^{2}$ ou $\pm 5 \%$ \\
\hline
\end{tabular}

Paralelamente ao monitoramento das variáveis climáticas, foram aplicados questionários de conforto, conforme a ISO 10551 (INTERNATIONAL..., 1995), envolvendo questões relativas à sensação térmica dos transeuntes. Foram levantados dados pessoais, tais como sexo, idade, altura, peso, local e tempo de moradia (aclimatação), vestimenta e tempo de exposição ao ambiente externo. Coletaram-se na sequência impressões dos transeuntes referentes à percepção térmica, avaliação e preferências térmicas, aceitação pessoal e tolerância. Para cada questão foi aplicada uma escala de intensidade, de acordo com as definidas pela ISO 10551 (INTERNATIONAL..., 1995).

Para a sensação térmica, analisada neste artigo, avaliaram-se as respostas à questão "Neste exato momento, você está sentindo...?”. As possibilidades de resposta estão no Quadro 1 (escala de percepção térmica).

Os valores de PET foram obtidos por meio do software RayMan (MATZARAKIS; RUTZ; MAYER 2010). Os dados de entrada são as variáveis climáticas, temperatura do ar, umidade relativa, temperatura radiante média e velocidade do ar. A taxa metabólica foi fixada em $165 \mathrm{~W} / \mathrm{m}^{2}$ (INTERNATIONAL..., 2004). Os dados pessoais utilizados para o cálculo do índice foram os valores de altura, peso e idade, referentes ao homem médio e à mulher média estabelecidos pela norma ISO 8896 (INTERNATIONAL..., 2004). Os procedimentos de calibração utilizam exclusivamente as respostas dos entrevistados quanto à sensação térmica versus os dados microclimáticos obtidos durante as campanhas de campo.

\section{Método 1}

Pelo primeiro método utilizado, as respostas de sensação térmica foram agrupadas da seguinte forma (ROSSI, 2012):

(a) desconforto para o frio: votos -3 e -2 ;

(b) conforto: votos $-1,0$ e +1 ; e (c) desconforto para o calor: $+2 \mathrm{e}+3$.

Foram comparados os votos agrupados às faixas de estresse térmico do PET, conforme apresentadas por Matzarakis e Mayer (1996) (Tabela 2). Para a calibração, os votos de conforto foram agrupados de acordo com o método proposto por De Dear e Fountain (1994), gerando-se uma média para cada 1 ${ }^{\circ} \mathrm{C}$ de variação na escala PET, ou seja, para cada intervalo foi obtida uma média dos votos de sensação térmica. Com o intuito de se reduzir a probabilidade de ocorrerem valores de sensação tendenciosos, adotou-se como critério de exclusão para a média agrupada de votos de sensação térmica um número mínimo de cinco respondentes por faixa de $1{ }^{\circ} \mathrm{C}$ (PET). A calibração das categorias de conforto/estresse térmico (Tabela 2) foi feita de acordo com a relação direta entre os valores médios de PET resultantes e o voto médio agrupado, por regressão linear.

Para cada categoria de estresse térmico, novas faixas foram definidas para tal índice, conforme a gradação da Tabela 2. A mesma tabela apresenta também a correspondência de faixas/PET em termos de variações do índice PMV, o qual representa a sensação térmica predita segundo uma escala de sete pontos com ponto neutro central (cf. Quadro 1). Complementarmente, foi calculada a porcentagem de insatisfeitos ("Percentage of Dissatisfied”, PD). Esse percentual de insatisfeitos PD foi comparado à equação sugerida por Fanger (1970) para a porcentagem predita de pessoas insatisfeitas ("Predicted Percentage Dissatisfied", PPD), conforme a norma ISO 7730 (INTERNATIONAL..., 2005), definindo-se também as faixas de PD para a amostra.

\section{Método 2}

Um segundo procedimento de calibração foi realizado com base no método proposto por Silva e Alvarez (2015), ao avaliarem o efeito da ventilação natural no conforto térmico ao nível do pedestre em Vitória, ES. A partir de dados coletados por meio de medições in loco e questionários aplicados a 841 respondentes, por três estações do ano (inverno, 
primavera e verão) em diferentes localizações a partir da costa, a calibração foi feita observando-se a predominância, isto é, a maior frequência de respostas térmicas segundo a escala de sete pontos com ponto neutro central (sensação térmica) de acordo com variações discretas de $1{ }^{\circ} \mathrm{C}$ na escala PET. O mesmo procedimento foi adotado por Lucchese et al. (2016) para Campo Grande, MT, com uma amostra menor, de 400 respondentes. Nesse estudo, realizado em uma praça pública, foram calibrados da mesma forma outros índices térmicos além do PET para inverno e primavera.

\section{Método 3}

O terceiro procedimento se baseia na aplicação do método Probit (MCFADDEN, $1973{ }^{3}$ apud GUJARATI, 1988). A expressão modelo Probit serve para designar modelos não lineares de probabilidade nos quais a função $\mathrm{F}$ obtida é uma distribuição de probabilidade (PINO, 2007). Esse método de regressão apresenta um modelo estatístico de dados ligados ao estímulo biológico, utilizado em estudos de conforto térmico para determinar a sensação térmica dos indivíduos em determinado nível de estímulo por meio de uma distribuição probabilística.

O Método de Regressão Probit determina probabilidades de duas situações de variáveis binárias, do tipo sim ou não, em relação a outras variáveis envolvidas. Para tanto, é necessário caracterizar a medição de cada variável climática e seus respectivos votos de sensação térmica, de modo a se definirem os limites de conforto térmico.

Segundo Xavier (1999), a presença de desconforto por calor em dada medição é constatada quando o percentual do somatório das pessoas que votaram, segundo a escala psicofísica de sete pontos, +3 ou "muito quente", +2 ou "quente" e $50 \%$ dos que votaram +1 "levemente quente" é superior a 30\% do total dos votos coletados na medição. Quando essa condição é verificada, diz-se que a variável dicotômica (em que só há duas respostas possíveis, no caso $0 / 1, \quad$ correspondendo a confortável/desconfortável) assume valor igual a 1, e a probabilidade de que $30 \%$ ou mais das pessoas encontrem-se desconfortáveis por calor é medida pela área abaixo da curva de distribuição normal. De maneira similar, é constatada a presença de desconforto por frio.

Quadro 1 - Questão analisada e escala adotada

\begin{tabular}{c|c|c|c|c|c|c}
\hline \multicolumn{2}{c|}{ Grau de intensidade } & Ponto neutro & \multicolumn{2}{c}{ Grau de intensidade } \\
\hline muito frio & frio & $\begin{array}{c}\text { um pouco } \\
\text { de frio }\end{array}$ & $\begin{array}{c}\text { neutralidade ou “nem } \\
\text { frio, nem calor” }\end{array}$ & $\begin{array}{c}\text { um pouco } \\
\text { de calor }\end{array}$ & calor & muito calor \\
\hline-3 & -2 & -1 & 0 & +1 & +2 & +3 \\
\hline
\end{tabular}

Tabela 2 - Sensação térmica e nível de estresse térmico para os índices PMV e PET

\begin{tabular}{|c|c|c|c|}
\hline PMV & PET & Sensacão térmica & Nível de estresse térmico \\
\hline & & muito frio & extremo estresse para o frio \\
\hline \multirow[t]{2}{*}{$-3,5$} & $4^{\circ} \mathrm{C}$ & |-------------------------------- & 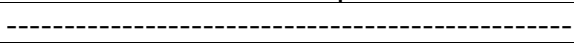 \\
\hline & & frio & forte estresse para o frio \\
\hline \multirow[t]{2}{*}{$-2,5$} & $8^{\circ} \mathrm{C}$ & |-------------------------------- & ---------------------------------------------------- \\
\hline & & pouco frio & moderado estresse para o frio \\
\hline \multirow[t]{2}{*}{$-1,5$} & $13^{\circ} \mathrm{C}$ & -------------------------------- & --------------------------------------------------------- \\
\hline & & levemente frio & leve estresse para o frio \\
\hline \multirow[t]{2}{*}{$-0,5$} & $18^{\circ} \mathrm{C}$ & |------------------------------- & ------------------------------------------------------- \\
\hline & & confortável & sem estresse térmico \\
\hline \multirow[t]{2}{*}{0,5} & $23^{\circ} \mathrm{C}$ & |-------------------------------- & |----------------------------------------------------- \\
\hline & & levemente calor & leve estresse para o calor \\
\hline \multirow[t]{2}{*}{1,5} & $29^{\circ} \mathrm{C}$ & -------------------------------- & ---------------------------------------------------- \\
\hline & & pouco calor & moderado estresse para o calor \\
\hline \multirow[t]{2}{*}{2,5} & $35^{\circ} \mathrm{C}$ & 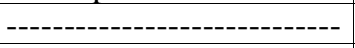 & ------------------------------------------------------- \\
\hline & & calor & forte estresse para o calor \\
\hline \multirow[t]{2}{*}{3,5} & $41^{\circ} \mathrm{C}$ & --------------------------------- & ------------------------------------------------------ \\
\hline & & muito calor & extremo estresse para o calor \\
\hline
\end{tabular}

Fonte: adaptado de Matzarakis e Mayer (1996).

${ }^{3}$ MCFADDEN, D. Conditional Logit Analysis of Qualitative Choice Behavior. Frontiers in Ecnometrics. New York: Academic Press, 1973. 
Pesquisas de campo que utilizam o Método de Regressão Probit foram anteriormente realizadas para prever a sensação térmica dos usuários em diversas cidades brasileiras, como os trabalhos de Araújo (1996), Xavier (1999), Gonçalves (2002) e Cristeli (2017).

No caso do presente estudo, adotaram-se três diferentes percentuais de insatisfeitos para a situação-limite da faixa de conforto, quais sejam, $30 \%$, $20 \%$ e $10 \%$, cujos resultados são posteriormente comparados. Tais percentuais iniciam-se proximamente à porcentagem de insatisfeitos mínima obtida pelo primeiro método, conforme apresentado a seguir.

\section{Análise de resultados}

A amostra final resultou em 1.685 questionários válidos. Em relação aos dados pessoais, tem-se uma amostra bem diversificada, que abrange diversas faixas etárias, características antropométricas e condições de isolamento térmico da vestimenta. $\mathrm{Na}$ Tabela 3 apresentam-se alguns dados biométricos da amostra, com classificação de idade e IMC segundo a Organização Mundial de Saúde (OMS).

Na Tabela 4 apresenta-se a análise descritiva dos dados climáticos monitorados em Curitiba. Os levantamentos abrangeram uma grande variedade de condições climáticas para todas as variáveis. Em relação à temperatura do ar, a mais baixa temperatura registrada foi de $6,4{ }^{\circ} \mathrm{C}$, e a mais alta, de $32,6{ }^{\circ} \mathrm{C}$.
A amostra de dados pessoais pode ser caracterizada por sexo, idade, altura, peso e vestimenta. Também se observa uma grande diversidade na amostra, com diferentes características físicas e condições de isolamento térmico, conforme dados apresentados na Tabela 5.

\section{Calibração do índice PET pelo método 1}

Optou-se por realizar a calibração somente para as faixas de desconforto/estresse térmico do índice PET que apresentassem a maior porcentagem de respostas. Foram calibradas as faixas de leve estresse térmico para o frio $(22 \%$ do total de respostas), sem estresse térmico (21\%) e leve estresse para o calor (20\%), ou seja, 63\% do total. Na Figura 2 mostra-se o gráfico de dispersão com as curvas de tendência e equações de regressão para os dados agrupados por intervalos de $1{ }^{\circ} \mathrm{C}$ na escala PET. Os valores referenciais de mudança de faixa de conforto/estresse térmico do PET (conforme a Tabela 2) também foram plotados (linha tracejada).

Verifica-se que a inclinação da curva de tendência difere visivelmente das faixas originais do índice PET. A neutralidade térmica ocorre em $19,2{ }^{\circ} \mathrm{C}$, havendo uma diferença de $0,6{ }^{\circ} \mathrm{C}$ em relação ao valor PET referencial para essa situação, que é de 18, $6^{\circ} \mathrm{C}$. A partir da Figura 2 é possível traçar faixas calibradas de estresse térmico (Tabela 6) aplicandose a equação de regressão encontrada.

Tabela 3 - Dados antropométricos das amostras de Curitiba

\begin{tabular}{l|l|c|c}
\hline \multicolumn{1}{c|}{ Critério } & \multicolumn{1}{|c|}{ Categorias } & Curitiba (N) & Curitiba (\%) \\
\hline \multirow{2}{*}{ Gênero } & Masculino & 967 & 57 \\
& Feminino & 718 & 43 \\
\hline \multirow{3}{*}{ Idade } & Até 25 anos ('jovem’) & 501 & 30 \\
& Entre 25 e 64 anos ('adulto') & 1.032 & 61 \\
& Acima de 64 anos ('idoso') & 152 & 9 \\
\hline \multirow{5}{*}{ Índice de Massa Corporal } & 62 & 4 \\
& Abaixo do peso (IMC $\left.<18,5 \mathrm{~kg} / \mathrm{m}^{2}\right)$ & 891 & 53 \\
& Normal $\left(18,5 \mathrm{~kg} / \mathrm{m}^{2} \leq \mathrm{IMC} \leq 24,5 \mathrm{~kg} / \mathrm{m}^{2}\right)$ & 544 & 32 \\
& Acima do peso $\left(\mathrm{IMC} \geq 25 \mathrm{~kg} / \mathrm{m}^{2}\right)$ & 188 & 11 \\
\hline
\end{tabular}

Tabela 4 - Análise descritiva das variáveis climáticas monitoradas

\begin{tabular}{|c|c|c|c|c|c|}
\hline & Va $(\mathrm{m} / \mathrm{s})$ & $\mathrm{RS}\left(\mathrm{W} / \mathbf{m}^{2}\right)$ & $\mathrm{Ta}\left({ }^{\circ} \mathrm{C}\right)$ & UR (\%) & $\operatorname{Trm}\left({ }^{\circ} \mathrm{C}\right)$ \\
\hline Média & 1,0 & 280 & 19,9 & 56 & 31,3 \\
\hline Desvio padrão & 0,5 & 302 & 5,5 & 13 & 13,9 \\
\hline Mínimo & 0,0 & 1 & 6,4 & 23 & 9,8 \\
\hline Máximo & 2,7 & 1.199 & 30,7 & 93 & 72,3 \\
\hline
\end{tabular}


Tabela 5 - Análise descritiva dos dados pessoais coletados

\begin{tabular}{l|c|c|c|c|c}
\hline & Idade (anos) & Altura (cm) & Peso (kg) & Peso (IMC) & clo \\
\hline Média & 38 & 169 & 71 & 25 & 0,7 \\
Desvio padrão & 17 & 9 & 14 & 4 & 0,3 \\
Mínimo & 13 & 130 & 40 & 16 & 0,2 \\
Máximo & 91 & 202 & 180 & 56 & 2,6 \\
\hline
\end{tabular}

Figura 2 - Votos médios de sensação térmica agrupada (ST) e curva de regressão

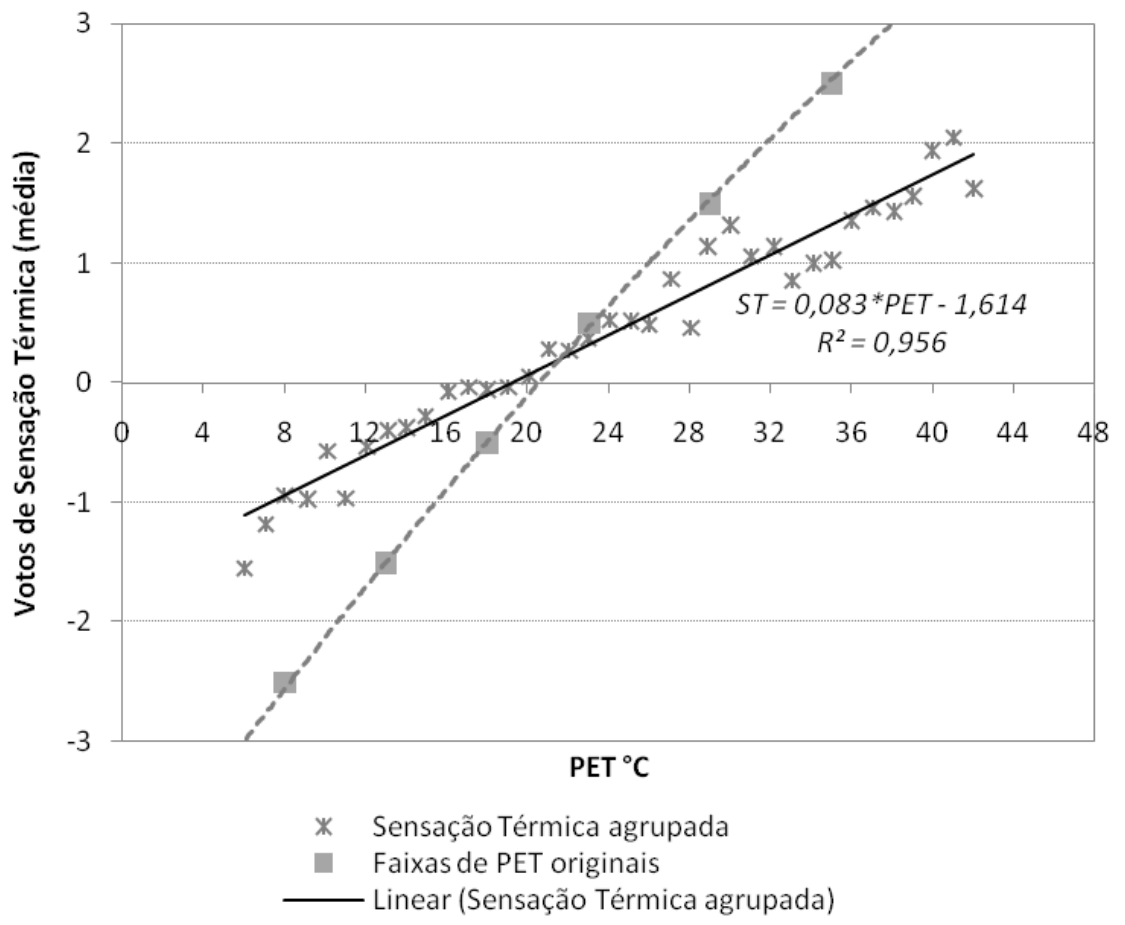

Na Tabela 6 apresentam-se os dados calibrados para Curitiba, as faixas originais do PET e sua correspondência com a escala de sete pontos proposta por Fanger (1970). A análise mostra que a população tem uma tolerância térmica maior para baixas temperaturas do que a proposta para o PET, visto que a temperatura equivalente em grau PET a "leve desconforto para o frio" reduziu-se em $5{ }^{\circ} \mathrm{C}$ em relação ao limite máximo original $\left(13^{\circ} \mathrm{C}\right.$ versus $\left.18{ }^{\circ} \mathrm{C}\right)$. Da mesma forma, parece haver maior tolerância para estresse térmico por calor/faixa de "leve estresse térmico para o calor”, que atingiu uma diferença de $8{ }^{\circ} \mathrm{C}$ em relação ao limite máximo proposto para tal faixa $\left(29{ }^{\circ} \mathrm{C}\right.$ versus $\left.37^{\circ} \mathrm{C}\right)$. A amplitude da faixa de neutralidade térmica foi aumentada em 7 graus PET. Tal aumento de “tolerância térmica” é verificado graficamente com a menor inclinação da reta de regressão (cf. Figura 2).

As porcentagens de insatisfeitos (PD) estão apresentadas na Figura 3, sobre a qual também se plotaram resultados da equação original para PPD ("Predicted Percentage Dissatisfied"), conforme a norma ISO 7730 (INTERNATIONAL..., 2005) para a faixa de sensação térmica analisada. O mínimo valor para PD foi de aproximadamente $12 \%$ (ou 11,64\%, obtidos a partir da equação apresentada na Figura 3, ao atribuir-se $\mathrm{x}=0$ para o "voto médio de sensação térmica”). A partir disso, a equação original para PPD foi ajustada para um mínimo de $12 \%$ PD (conforme o valor encontrado pela equação, em vez de 5\%, para ambientes internos com ar condicionado), considerando que em espaços abertos divergências em termos de sensação térmica reportada são maiores que em espaços fechados. A equação obtida por regressão polinomial e o ajuste na equação de PPD são apresentados na Tabela 7 .

As porcentagens obtidas pela equação ajustada apresentam maiores diferenças de valores na categoria "sem estresse térmico" (entre -0,5 e +0,5). A partir da equação polinomial plotada sobre a Figura 3, puderam ser obtidas as PD para cada categoria de estresse térmico (cf. Tabela 6). Para as categorias de "estresse térmico para o calor", os valores ajustados se aproximam dos valores originais propostos por Fanger (1970), com diferença de apenas 1\% (Tabela 6). 
Tabela 6 - Calibração de níveis de estresse térmico para o índice PET (valores em ํ) para Curitiba método 1

\begin{tabular}{c|c|c|l}
\hline $\begin{array}{c}\text { Escala de sete } \\
\text { pontos }\end{array}$ & $\begin{array}{c}\text { PET/PPD } \\
\text { original }\end{array}$ & $\begin{array}{c}\text { PET/PD } \\
\text { (Curitiba) }\end{array}$ & Nível de estresse térmico \\
\hline$-1,5$ & $13^{\circ} \mathrm{C}$ & - & \\
\hline & & & leve estresse para o frio \\
\hline$-0,5$ & $18^{\circ} \mathrm{C} / 10 \%$ & $13^{\circ} \mathrm{C} / 15 \%$ & \\
\hline 0,5 & & & sem estresse térmico \\
\hline & $23^{\circ} \mathrm{C} / 10 \%$ & $25^{\circ} \mathrm{C} / 17 \%$ & \\
\hline 1,5 & & & leve estresse para o calor \\
\hline & $29^{\circ} \mathrm{C} / 51 \%$ & $37^{\circ} \mathrm{C} / 50 \%$ & \\
\hline 2,5 & & & moderado estresse para o calor \\
\hline & $35^{\circ} \mathrm{C} / 93 \%$ & - & \\
\hline 3,5 & & & forte estresse para o calor \\
\hline & $41^{\circ} \mathrm{C}$ & - & \\
\hline & & & extremo estresse para o calor \\
\hline
\end{tabular}

Figura 3 - Porcentagem de insatisfeitos (PD) versus votos de sensação térmica médios agrupados, para Curitiba

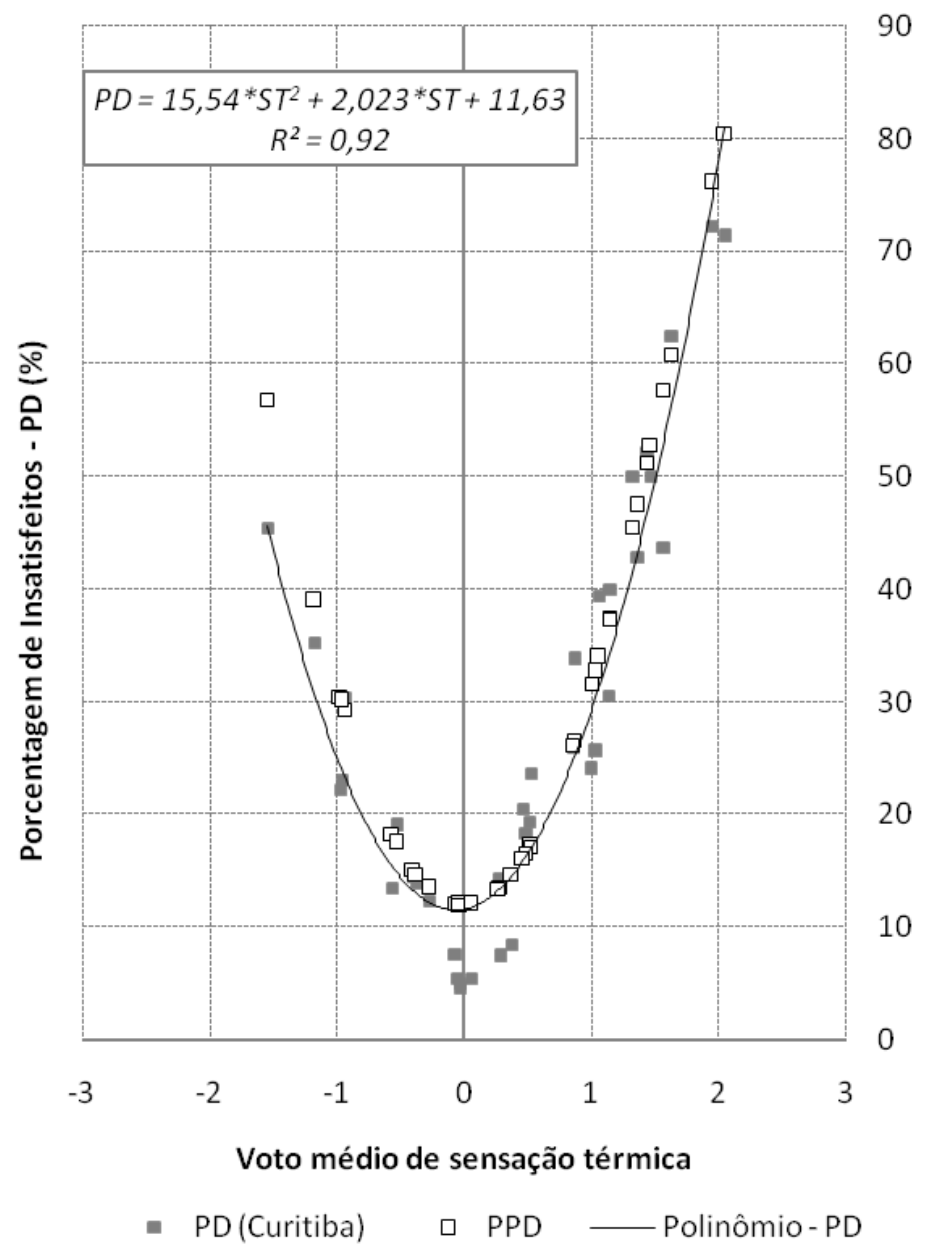

Tabela 7 - Equações de regressão para PD e equação ajustada de PPD

\begin{tabular}{c|c}
\hline $\begin{array}{c}\text { Equação obtida a partir de curva de } \\
\text { tendência (polinomial) }\end{array}$ & Equação ajustada de PPD para a amostra \\
\hline $\mathrm{PD}=15,54 * \mathrm{ST} \mathrm{T}^{2}+2,0231 * \mathrm{ST}+11,637$ & $P P D_{\text {ajustado }}=10-88 \cdot e^{-\left(0,03353 \cdot P M V^{4}+0,2179 . P M V^{2}\right)}$ \\
\hline
\end{tabular}




\section{Calibração do índice PET pelo método 2}

Realizando o mesmo procedimento de agrupar respostas de ST para um mínimo de 5 pessoas em cada variação de $1{ }^{\circ} \mathrm{C}$ na escala $\mathrm{PET}$, pode ser identificada a predominância de determinado voto de sensação térmica para cada variação de $1{ }^{\circ} \mathrm{C}$ na escala PET. Na Figura 4 apresentam-se os percentuais empilhados para cada voto, sendo demarcados no gráfico os mais altos percentuais de entrevistados em determinada classe de sensação térmica. No caso de duas classes com mesmo valor percentual, não é destacada a coluna predominante.
A predominância de votos de sensação térmica iguais a zero $(\mathrm{ST}=0)$, identificados claramente no gráfico (entre $12{ }^{\circ} \mathrm{C}$ e $28{ }^{\circ} \mathrm{C}$ na escala PET), permite comparações com as faixas de conforto térmico obtidas para Vitória e Campo Grande nos estudos relatados (SILVA; ALVAREZ, 2015; LUCCHESE et al., 2016), segundo a mesma metodologia (Tabela 8).

\section{Calibração do índice PET pelo método 3}

A aplicação do método Probit assumindo três diferentes percentuais de insatisfeitos para a situação-limite da faixa de conforto é apresentada nas Figuras 5 a 7 respectivamente.

Figura 4 - Percentuais empilhados de entrevistados por opção de voto de sensação térmica (ST) com contornos para percentuais dominantes, para Curitiba

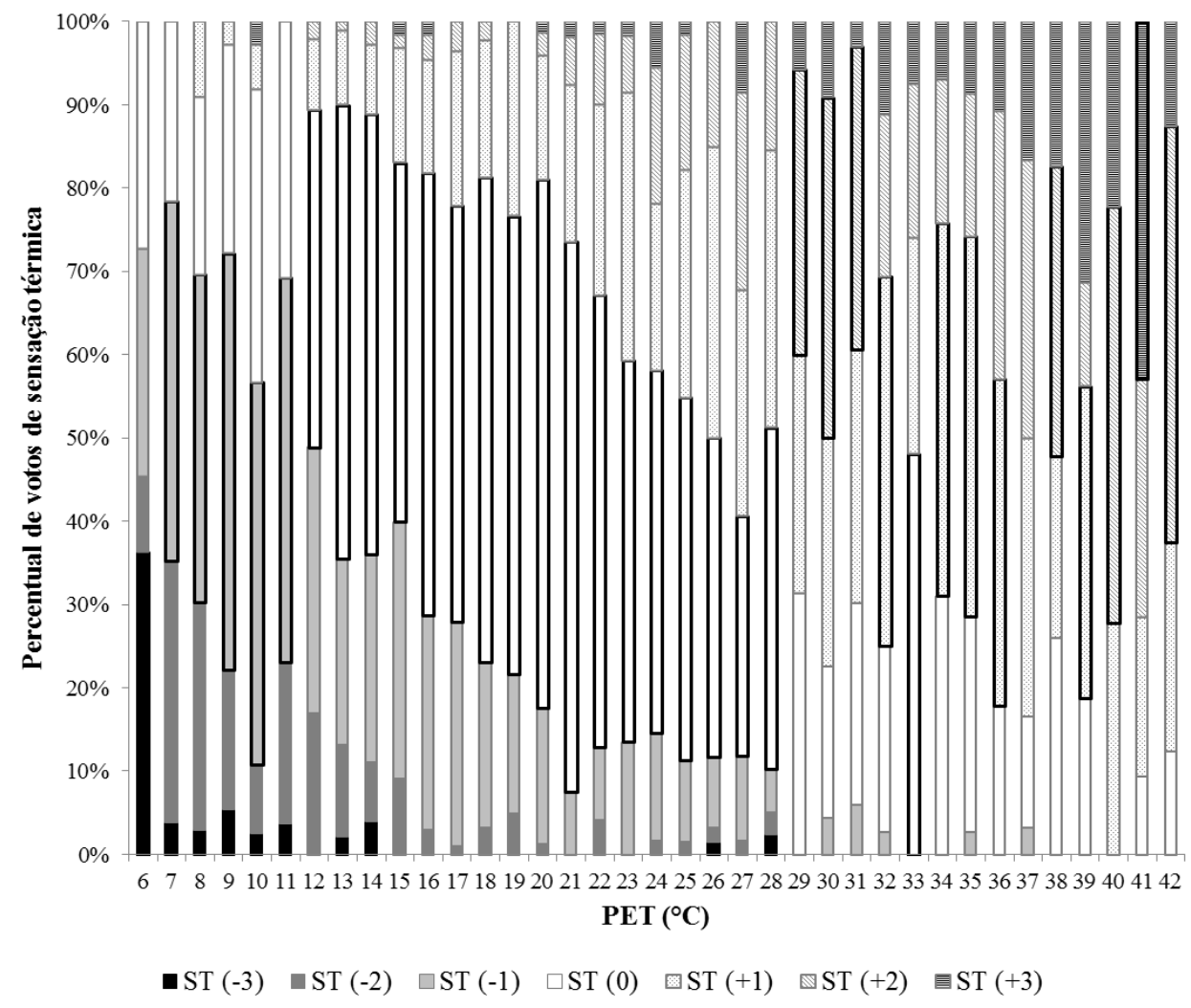

Tabela 8 - Faixas de conforto (ST=0) para o índice PET, para Curitiba, Vitória e Campo Grande - método 2

\begin{tabular}{c|c|c|c}
\hline Original & Curitiba & $\begin{array}{c}\text { Vitória } \\
\text { (Silva e Alvarez, 2015) }\end{array}$ & $\begin{array}{c}\text { Campo Grande } \\
\text { (Lucchese et al., 2016) }\end{array}$ \\
\hline $18^{\circ} \mathrm{C} \mathrm{a} 23^{\circ} \mathrm{C}$ & $12^{\circ} \mathrm{C} \mathrm{a} 28^{\circ} \mathrm{C}$ & $22^{\circ} \mathrm{C}$ a $30^{\circ} \mathrm{C}$ & $21^{\circ} \mathrm{C} \mathrm{a} 27^{\circ} \mathrm{C}$ \\
\hline
\end{tabular}


Figura 5 - Curvas Probit para frio (votos -3, -2 e 50\% dos votos - 1 ) e calor (votos $+3,+2$ e $50 \%$ dos votos $+1)$ com indicação da faixa de $10 \%$ de insatisfeitos

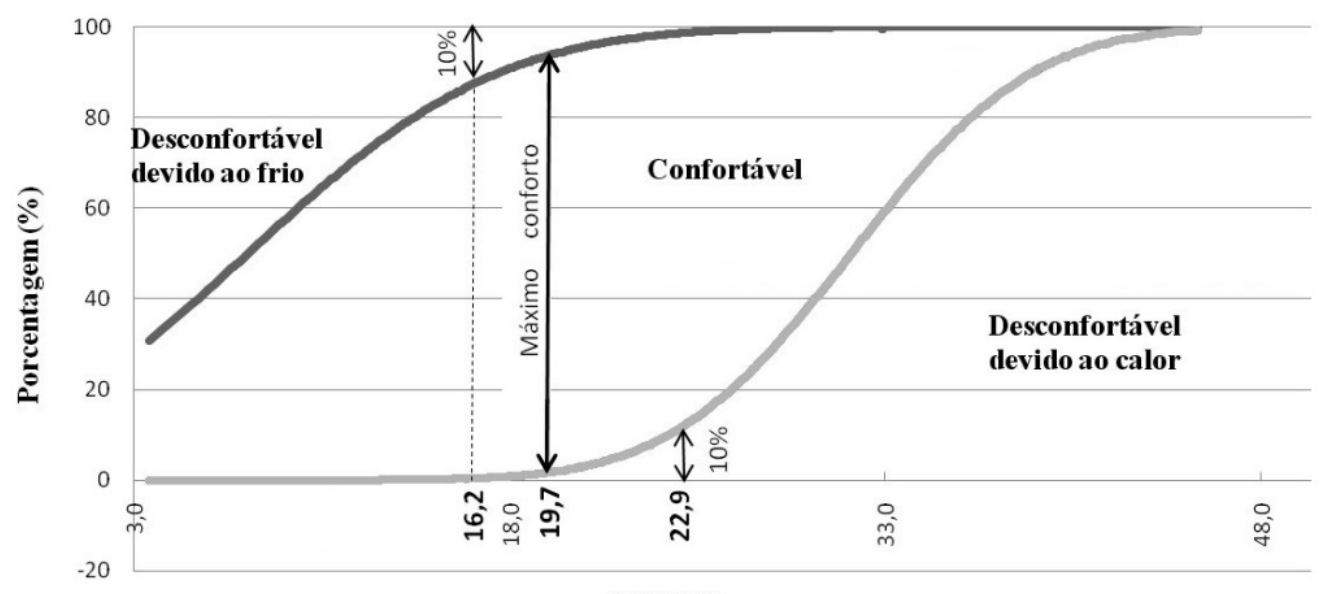

PET $\left({ }^{\circ} \mathrm{C}\right)$

Figura 6 - Curvas Probit para frio (votos -3, -2 e 50\% dos votos -1) e calor (votos $+3,+2$ e $50 \%$ dos votos $+1)$ com indicação da faixa de $20 \%$ de insatisfeitos

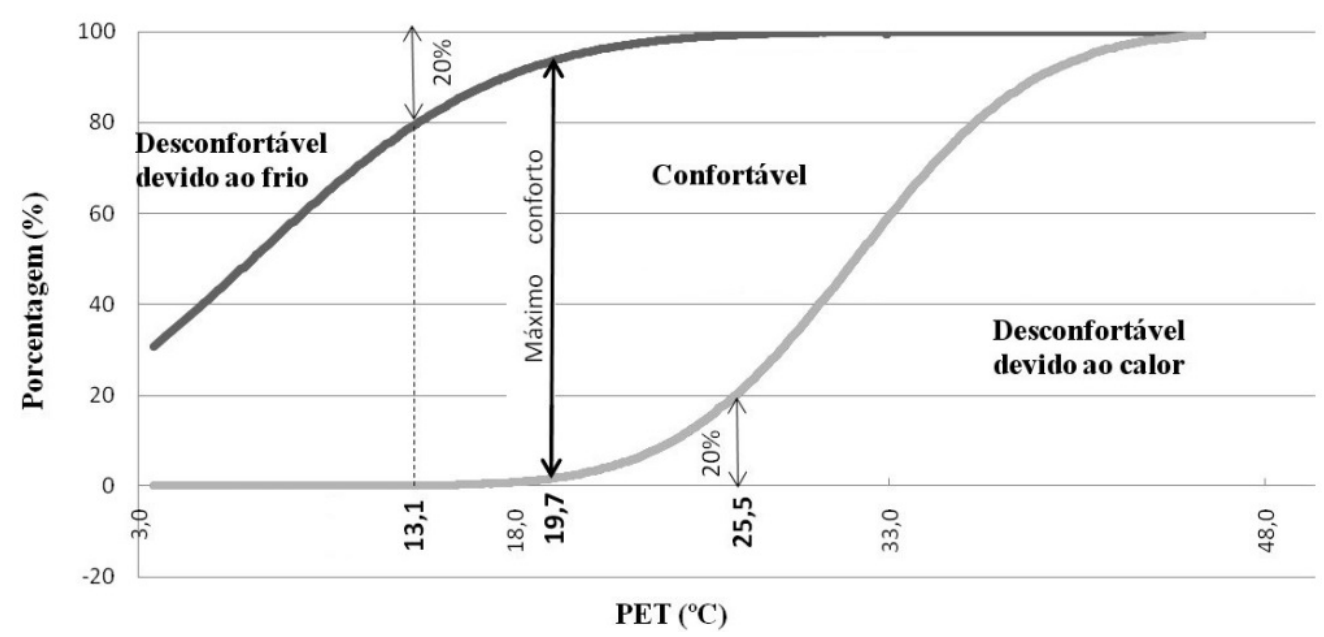

Figura 7 - Curvas Probit para frio (votos -3, -2 e $50 \%$ dos votos -1 ) e calor (votos $+3,+2$ e $50 \%$ dos votos $+1)$ com indicação da faixa de $50 \%$ de insatisfeitos

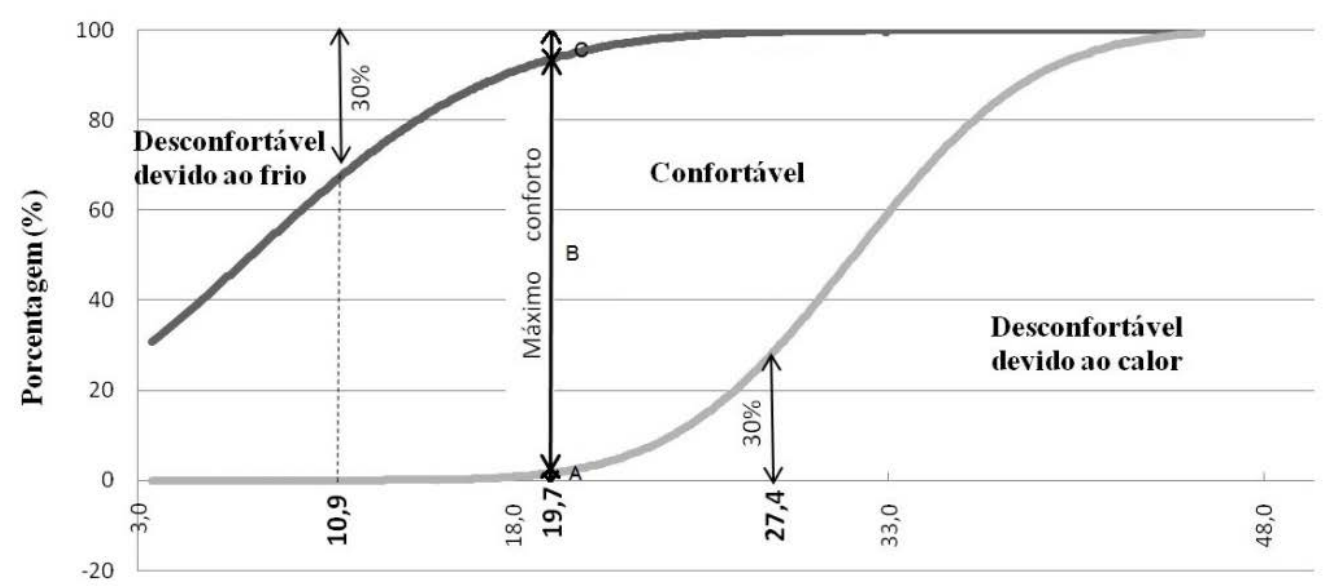

PET $\left({ }^{\circ} \mathrm{C}\right)$ 
A interpretação dos resultados das curvas Probit geradas é dada por Fontanella (2009). Uma linha traçada em algum valor do eixo das ordenadas (temperatura em PET), como no ponto de máximo conforto, de $19,7^{\circ} \mathrm{C}$, por exemplo, intercepta essas curvas formando três segmentos de reta, conforme representados didaticamente na Figura 7. O comprimento total da linha representa $100 \%$ dos votos do grau de satisfação, mas cada segmento tem um significado diferente: o segmento inferior A representa a porcentagem de escolha relativa ao desconforto devido ao calor; o segmento central, B, representa a porcentagem confortável; e o segmento superior $\mathrm{C}$ representa a porcentagem relativa ao desconforto devido ao frio.

Nas Figuras 5 a 7 podem-se notar faixas variáveis de conforto para o índice PET, dadas segundo a situação-limite considerada (10\%, 20\% e 30\% de insatisfeitos), permanecendo invariável o valor do índice PET com a maior percentagem de pessoas em situação de conforto, de $19,7{ }^{\circ} \mathrm{C}$, para $92,9 \%$ de pessoas satisfeitas com o ambiente térmico. $\mathrm{Na}$ Tabela 9 resumem-se os valores encontrados, sendo as faixas cada vez mais restritivas em função dos percentuais adotados de pessoas insatisfeitas.

\section{Discussão}

Uma verificação dos limites de conforto para os três métodos é apresentada na Tabela 10, onde a base de dados permitiu compor uma região de conforto para o índice PET. Nesse caso, apresentam-se valores médios para o índice PET para cada faixa definida, a mediana correspondente àquela faixa, assim como a porcentagem de acertos, ou seja, o percentual de votos equivalentes à categoria " 0 ” (neutralidade ou “nem frio, nem calor”, conforme o Quadro 1) para cada faixa. Na Tabela 10 são apresentados, a título de referência, os mesmos indicadores para a faixa original do índice PET (cf. Tabela 2).

A interpretação da Tabela 10 deve levar em conta a quantidade de respostas, a média de sensação térmica e o percentual de resposta de $\mathrm{ST}=0$ para cada faixa pela respectiva quantidade de respostas (percentual de acertos). Nota-se que o percentual de acertos aumenta quanto mais restrita for a faixa de conforto, porém acompanhado de pequeno aumento na média de sensação térmica na faixa. A mediana não sofre alteração em nenhum momento. As maiores faixas de conforto atingem percentuais de acertos mais baixos, podendo-se, dessa forma, descartar a faixa obtida pelo método 2 e pelo método 3 para $30 \%$ de pessoas insatisfeitas. As faixas obtidas pelo método 1 e pelo método $3 \mathrm{com}$ $20 \%$ de insatisfeitos são equivalentes e podem ser adotadas como padrão no caso de Curitiba.

Tal constatação corrobora resultados apresentados por Pantavou et al. (2014), que compararam o método de regressão linear a outros dois métodos na calibração de índices de conforto (incluindo o índice PET) para ambientes externos monitorados em zonas climáticas mediterrâneas, quais sejam: análise Probit e regressão cúbica. Na comparação, a análise Probit não apresentou resultados significativamente mais precisos do que os obtidos via regressão linear, tendo havido similaridade nos resultados.

Tabela 9 - Faixas de conforto para o índice PET, para Curitiba - método 3

\begin{tabular}{c|c}
\hline Percentual de insatisfeitos & Valores em PET \\
\hline $10 \%$ & $16,2^{\circ} \mathrm{C}$ a $22,9{ }^{\circ} \mathrm{C}$ \\
$20 \%$ & $13,1^{\circ} \mathrm{C}$ a $25,5^{\circ} \mathrm{C}$ \\
$30 \%$ & $10,9^{\circ} \mathrm{C}$ a $27,4{ }^{\circ} \mathrm{C}$ \\
\hline
\end{tabular}

Tabela 10 - Comparativo entre faixas de conforto para o índice PET, para Curitiba, pelos três métodos analisados

\begin{tabular}{l|c|c|c|c|c|c}
\hline \multicolumn{1}{c|}{$\begin{array}{c}\text { Procedimento de } \\
\text { calibração }\end{array}$} & Faixa & $\mathbf{n}$ & Média (ST) & $\begin{array}{c}\text { Mediana } \\
\text { (ST) }\end{array}$ & n votos "0" & \% acertos \\
\hline Faixa original (Tabela 2) & $18^{\circ} \mathrm{C} \mathrm{a} 23^{\circ} \mathrm{C}$ & 343 & 0,1 & 0 & 203 & $59 \%$ \\
\hline Método 1 & $13^{\circ} \mathrm{C}$ a $25^{\circ} \mathrm{C}$ & 839 & 0,0 & 0 & 442 & $53 \%$ \\
\hline Método 2 & $12^{\circ} \mathrm{C} \mathrm{a} 28^{\circ} \mathrm{C}$ & 1.068 & 0,1 & 0 & 537 & $50 \%$ \\
\hline $\begin{array}{l}\text { Método 3 } 10 \% \text { de } \\
\text { insatisfeitos }\end{array}$ & $16,2^{\circ} \mathrm{C} \mathrm{a} 22,9^{\circ} \mathrm{C}$ & 494 & 0,1 & 0 & 286 & $58 \%$ \\
\hline $\begin{array}{l}\text { Método 3 - 20\% de } \\
\text { insatisfeitos }\end{array}$ & $13,1^{\circ} \mathrm{C} \mathrm{a} 25,5^{\circ} \mathrm{C}$ & 868 & 0,0 & 0 & 456 & $53 \%$ \\
\hline $\begin{array}{l}\text { Método 3- 30\% de } \\
\text { insatisfeitos }\end{array}$ & $10,9^{\circ} \mathrm{C}$ a $27,4^{\circ} \mathrm{C}$ & 1.078 & 0,0 & 0 & 542 & $50 \%$ \\
\hline
\end{tabular}

Calibração do índice de conforto para espaços externos Physiological Equivalent Temperature (PET) para Curitiba 
A discrepância entre a faixa de conforto obtida pelo método 1 e aquela obtida pelo método 2 pode ser explicada pela definição pouco nítida dos limites inferior e superior em cada faixa de sensação térmica por este último método. Exemplificando, a faixa imediatamente superior $(\mathrm{ST}=+1)$ apresenta um salto inicial de duas categorias (de $\mathrm{ST}=0$ a $\mathrm{ST}=+2$ ), assumindo então $\mathrm{ST}=+1$, voltando à condição de neutralidade e, por fim, chegando novamente a $\mathrm{ST}=+1$ (Figura 2 ).

O percentual de insatisfeitos a ser considerado para definição de limites no método 3 , em espaços externos, a partir da análise anterior da porcentagem de insatisfeitos (PD) (conforme Figura 3), parece situar-se em torno de $20 \%$, uma vez que a correspondência com ST seria para $-0,5$ e +0,5 (conforme a definição das curvas Probit, qual seja, "frio" = votos -3 , -2 e $50 \%$ dos votos -1 ; "calor" = votos +3 , +2 e $50 \%$ dos votos +1 ). Estudo anterior visando à calibração do índice térmico "Universal Thermal Climate Index” (UTCI) para Curitiba mostrou valores semelhantes de pessoas insatisfeitas (ROSSI; KRÜGER; BRÖDE, 2012). Como neste estudo, obteve-se que o limite mínimo da zona de conforto é inferior ao sugerido pelos desenvolvedores do índice PET. O motivo disso pode estar associado à maior exposição ao frio por moradores de Curitiba, o que faz com que os moradores desenvolvam aclimatação térmica às condições climáticas locais. Ressalta-se que o uso intensivo de calefação ou de aquecimento artificial ainda é baixo nessa cidade, o que não ocorre na Europa, para onde foi desenvolvido o índice.

\section{Conclusões}

Como resultados, observaram-se semelhanças entre dois métodos utilizados, sendo o percentual a ser adotado no método Probit relevante na correta definição da zona de conforto para Curitiba. A utilização dos dois métodos (regressão linear de dados agrupados e análise Probit) deveria ocorrer, dessa forma, de modo complementar, uma vez que se pode corroborar a faixa definida no primeiro método e, na análise Probit, se adotar com mais propriedade o percentual de insatisfeitos a ser considerado como situação-limite (“cut-offs”) para a zona de conforto térmico. Os resultados apresentados reforçam a necessidade de calibração de índices como o PET para outras localidades, com realidades climáticas e populações com percepções térmicas diversas às das faixas originalmente propostas pelos desenvolvedores de tais índices térmicos.

Iniciativas futuras de aprimoramento envolvem, por exemplo, a calibração do índice de conforto para espaços abertos UTCI a partir de análise Probit, de modo a comprovar a semelhança entre os métodos de regressão linear e curvas Probit, observada para o índice PET a partir da mesma base de dados. Nesse caso, o estudo seria uma extensão dos resultados de calibração anterior realizada para o UTCI (ROSSI; KRÜGER; BRÖDE, 2012).

\section{Referências}

ALI-TOUDERT, F.; MAYER, H. Effects of Street Design on Outdoor Thermal Comfort. In: KISS, A.; MEZÖSI, G.; SÜMEGHY, Z. (Eds.).

Landscape, Environment and Society. Studies in Honour of Professor Ilona Bárány-Kevei on the Occasion of her Birthday. SZTE Éghajlattani és Tájföldrajzi Tanszék, Természeti Földrajzi és Geoinformatikai Tanszék, Szeged, 2006.

ARAÚJO, V. M. D. Parâmetros de Conforto Térmico Para Usuários de Edificações Escolares no Litoral Nordestino Brasileiro. São Paulo, 1996. Tese (Doutorado em Arquitetura e Urbanismo) - Faculdade de Arquitetura e Urbanismo, Universidade de São Paulo, São Paulo, 1996.

\section{ASSOCIATION OF GERMAN ENGINEERS.} VDI 3787: environmental meteorology: methods for the human biometeorological evaluation of climate and air quality for urban and regional planning at regional level: part I: climate, blatt 2/ part 2, 2008.

CRISTELI, P. S. Obtenção de Zona de Conforto Utilizando a Regressão PROBIT e o Método de Médias Associadas Para as Condições

Climáticas de Ouro Preto: ambientes universitários. Ouro Preto, 2017. Dissertação (Mestrado em Engenharia Civil) - Escola de Minas, Universidade Federal de Ouro Preto, Ouro Preto, 2017.

DE DEAR, R. J.; FOUNTAIN, M. E. Thermal Comfort in Air Conditioned Office Buildings in the Tropics. Journal of the Australian Institute of Refrigerating, Air-Conditioning and Heating, v. 48, n. 9, p. 14-30, 1994.

FANGER, P. O. Thermal Comfort: analysis and applications in environmental engineering. New York: McGraw, 1970.

FONTANELLA, M. S. Percepção do Ambiente Térmico: preferências subjetivas e conforto térmico. Ouro Preto, 2009. Dissertação (Mestrado em Engenharia Civil) - Programa de PósGraduação de Engenharia Civil, Escola de Minas, Universidade Federal de Ouro Preto, Ouro Preto, 2009. 
GONÇALVES, W. B. Estudo de Índices de Conforto Térmico Avaliados Com Base em População Universitária na Região Metropolitana de Belo Horizonte. Belo Horizonte, 2002. Dissertação (Mestrado em Engenharia Mecânica) - Escola de Engenharia, Universidade Federal de Minas Gerais, Belo Horizonte, 2002.

GUJARATI, D. N. Basic Econometrics. New York: McGraw-Hill, 1988.

GUJARATI, D. N. Basic Econometrics. New York: McGraw-Hill, 1988.

HIRASHIMA, S. et al. Thermal comfort comparison and evaluation in different climates In: INTERNATIONAL CONFERENCE ON URBAN CLIMATE, 9., Toulouse, 2015. Proceedings... Toulouse, 2015.

HÖPPE, P. The Physiological Equivalent Temperature: an index for the biometeorological assessment of the thermal environment.

International Journal of Biometeorology, v. 43. p. 71-75, 1999.

INTERNATIONAL ORGANIZATION FOR STANDARDIZATION. ISO 10551: ergonomics of the thermal environments: assessment of the influence of the thermal environment using subjective judgment scales. Genebra, 1995.

INTERNATIONAL ORGANIZATION FOR STANDARDIZATION. ISO 7726: ergonomics of the thermal environments: instruments for measuring physical quantities. Genebra, 1998.

INTERNATIONAL ORGANIZATION FOR STANDARDIZATION. ISO 7730: ergonomics of the thermal environments: analytical determination and interpretation of thermal comfort using calculation of the PMV and PPD indices and local thermal comfort criteria. Genebra, 2005.

INTERNATIONAL ORGANIZATION FOR STANDARDIZATION. ISO 8896: ergonomics of the thermal environments: determination of thermal metabolic rate. Genebra, 2004.

JOHANSSON, E. et al. Instruments and Methods in Outdoor Thermal Comfort Studies: the need for standardization. Urban Climate, v. 10, p. 346366, 2014.

JOHANSSON, E. Influence of Urban Geometry on Outdoor Thermal Comfort in a Hot Dry Climate: a study in Fez, Morocco. Building and Environment, v. 41, p. 1326-1338, 2006.
JOHANSSON, E.; EMMANUEL, R. The Influence of Urban Design on Outdoor Thermal Comfort in the Hot, Humid city of Colombo, Sri Lanka. International Journal of Biometeorology, v. 51, p. 119-133, 2006.

LAI, D. et al. Studies of Outdoor Thermal Comfort in Northern China. Building and Environment, v. 77, p. 110-118, 2014.

LIN, T. P.; MATZARAKIS, A. Tourism Climate and Thermal Comfort in Sun Moon Lake, Taiwan. International Journal of Biometeorology, v. 52, p. 281-290, 2008.

LOIS, E.; LABAKI, L. C. Conforto Térmico em Espaços Externos: uma revisão. In: ENCONTRO NACIONAL DE CONFORTO NO AMBIENTE CONSTRUÍDO, 6., São Pedro, 2001. Anais... São Pedro, 2001.

LUCCHESE, J. R. et al. Application of Selected Indices on Outdoor Thermal Comfort Assessment in Midwest Brazil. International Journal of Science and Engineering Investigations, v. 7, n. 4, p. 291-302, 2016.

MAKAREMI, N. et al. Thermal Comfort Conditions of Shaded Outdoor Spaces in Hot and Humid Climate of Malaysia. Building and Environment, v. 48, p. 7-14, 2011.

MATZARAKIS, A.; BLAZEJCZYK, K.; AMELUNG, B. Climate Change and Tourism Assessment and Coping Strategies. Freiburg: Maastricht-Warsaw, 2007.

MATZARAKIS, A.; MAYER, H. Another Kind of Environmental Stress: thermal stress. WHO Newsletter, v. 18, p. 7-10, 1996.

MATZARAKIS, A.; RUTZ, F.; MAYER, H. Modelling Radiation Fluxes in Simple and Complex Environments: basics of the RayMan model. International Journal of Biometeorology, v. 54, p. 131-139, 2010.

MONTEIRO, L. M.; ALUCCI, M. P. Calibration of Outdoor Thermal Comfort Models. In: CONFERENCE ON PASSIVE AND LOW ENERGY ARCHITECTURE, 23., Genebra, 2006. Proceedings... Genebra, 2006.

PANTAVOU, K. et al. Empirical Calibration of Thermal Indices in an Urban Outdoor Mediterranean Environment. Building and Environment, v. 8, p. 283-292, 2014.

PINO, F. A. Modelos de Decisão Binários. Revista de Economia Agrícola, v. 54, n. 1, p. 4357, 2007. 
ROSSI, F. A. Proposição de Metodologia e de Modelo Preditivo Para Avaliação de Sensação Térmica em Espaços Abertos em Curitiba. Curitiba, 2012. Tese (Doutorado em Tecnologia) Universidade Tecnológica Federal do Paraná, Curitiba, 2012.

ROSSI, F. A.; KRÜGER, E. L.; BRÖDE, P. Definição de Faixas de Conforto e Desconforto Térmico Para Espaços Abertos em Curitiba, PR, Com o Índice UTCI. Ambiente Construído, Porto Alegre, v. 12, n. 1, p. 41-59, jan./mar. 2012.

ROSSI, F. A.; KRÜGER, E. L.; BRÖDE, P. Definição de Faixas de Estresse Térmico Para Espaços Abertos em Curitiba, Paraná. In: ENCONTRO NACIONAL DE CONFORTO NO AMBIENTE CONSTRUÍDO, 11., Búzios, 2011. Anais... Búzios: ANTAC, 2011.

ROSSI, F. A.; KRÜGER, E. L.; NIKOLOPOULOU, M. A Influência da Configuração Urbana no Microclima e Sensação Térmica em Ruas de Pedestre de Curitiba, Paraná. In: ENCONTRO NACIONAL DE CONFORTO NO AMBIENTE CONSTRUÍDO, 11., Búzios, 2011. Anais... Búzios: ANTAC, 2011.
SALATA, F. et al. Outdoor Thermal Comfort in the Mediterranean Area: a transversal study in Rome, Italy. Building and Environment, v. 96, p. 46-61, 2016.

SILVA, F. T.; ALVAREZ, C. E. de. An Integrated Approach For Ventilation's Assessment on Outdoor Thermal Comfort. Building and Environment, v. 87, p. 59-71, 2015.

THORSSON, S. et al. Thermal Comfort and Outdoor Activity in Japanese Urban Public and Places. Environmental Behavior, v. 39, p. 660684, 2007.

\section{XAVIER, A. A. P. Condições de Conforto} Térmico Para Estudantes de $2^{\circ}$ Grau na Região de Florianópolis. Florianópolis, 1999. Dissertação (Mestrado em Engenharia Civil) - Escola de Engenharia, Universidade Federal de Santa Catarina, Florianópolis, 1999.

\section{Eduardo Leite Krüger}

Departamento de Construção Civil | Universidade Tecnológica Federal do Paraná | Rua Deputado Heitor Alencar Furtado, 4900, Campus Curitiba - Sede Ecoville | Curitiba - PR - Brasil | CEP 81280-340 | Tel.: (41) 3279-6821 | E-mail: ekruger@utfpr.edu.br

\section{Francine Aidie Rossi}

Departamento de Expressão Gráfica | Universidade Federal do Paraná | Av. Cel. Francisco H. dos Santos, 100, Centro Politécnico, J ardim das Américas | Curitiba - PR - Brasil | CEP 81530-000 | Tel.: (41) 3361-3462 | E-mail: rossi@ufpr.br

\section{Pablyne Sant' Ana Cristeli}

Programa de Pós-Graduação em Engenharia Civil | Universidade Federal de Ouro Preto | Campus Morro do Cruzeiro | Ouro Preto - MG Brasil | CEP 35400-000 | Tel.: (31) 98941-0420 | E-mail: paycristeli@hotmail.com

\section{Henor Artur de Souza}

Departamento de Engenharia de Controle e Automação e Técnicas Fundamentais, Escola de Minas | Universidade Federal de Ouro Preto | Tel.: (31) 3559-1533 Ramal 1482 | E-mail: henorster@gmail.com

Revista Ambiente Construído

Associação Nacional de Tecnologia do Ambiente Construído

Av. Osvaldo Aranha, 99 - 30 andar, Centro

Porto Alegre - RS - Brasil CEP $90035-190$

Telefone: +55 (51) 3308-4084 Fax: +55 (51) 3308-4054

www. seer. ufrgs. br/ ambienteconstruido

E-mail: ambienteconstruido@ufrgs.br 